\title{
Collapsing Layers on Schwarzschild-Lemaitre Geodesics
}

\author{
J.P. Krisch and E.N. Glass \\ Department of Physics, University of Michigan, Ann Arbor, MI 48109
}

(Dated: 5 September 2007)

\begin{abstract}
We discuss Israel layers collapsing inward from rest at infinity along SchwarzschildLemaitre geodesics. The dynamics of the collapsing layer and its equation of state are developed. There is a general equation of state which is approximately polytropic in the limit of very low pressure. The equation of state establishes a new limit on the stress-density ratio.

PACS numbers: 04.40. -b, 04.20.Jb
\end{abstract}

\section{INTRODUCTION}

Since their introduction, Israel layers [1], [2], [3] have played an increasingly important role in gravitational physics. Barrabes and Israel [3] began their paper with a description of the Israel layer as a thermodynamic phase boundary, but the initial applications of Israel layers considered metric matching in dynamic collapse processes involving dust shells, null shells, and cosmic string loops [1], 22],[3]. Poisson [4] has summarized some of the early seminal work by Israel [1],[2], Barrabes [3], de la Cruz [5], Musgrave and Lake [6], and Barrabes and Hogan [7]. The Israel junction conditions [1],22] find wide application because they provide a simple dynamic boundary description for a variety of scenarios [8]. The use of layers as boundaries between standard metrics has been summarized by Ansoldi [9], and there is a growing literature that applies the thin shell formalism to new areas such as

shell quantum mechanics [9] - [12], layer dimensionality [13], 14], (15], layers in extensions of general relativity [16] - 19] and matching in perturbed spacetimes [20] - [24]. Astrophysical problems using layers with constant spacetime character continue to be interesting, and include the investigation of collapse [25], 26], [27] and new phenomena such as gravastars [28] - [35]. A review of recent layer applications reveals increasingly complex layer models, the gravastar models, for example, involving multi-layer constructions, and there is an increasing interest in describing more realistic layers [36], [37]. 
A layer metric is determined by the metrics of the two bounding manifolds. The stressenergy of the layer is determined by jumps in the extrinsic curvatures of the metrics on either side of the layer. For example, the Israel layer between exterior vacuum Schwarzschild and interior Minkowski has a $2+1$ metric as seen from both sides of the layer [4]

$$
d s^{2}=-d \tau^{2}+R^{2}(\tau) d \Omega^{2} .
$$

The development of $R(\tau)$ tracks the dynamics of the layer and, as in any general relativity solution with stress-energy, the equation of state of the layer is important in characterizing the dynamics.

In this work we discuss layers which start from rest at infinity in an exterior Schwarzschild metric with mass parameter $m_{0}$ and drop inward along a geodesic. The Lemaitre form of the Schwarzschild metric is adapted to this particular geodesic motion but the layer motion can be simply described with the usual Schwarzschild metric. The interior space is Schwarzschild with mass parameter $M$. Although the motion of the layer is simple, the equation of state is cubic in the stress, quartic in the density and it provides a new restriction on the range of the stress/density ratio for collapsing layers.

Layers with simple motions, such as the layer considered here, can require more physical content than the density and pressure coming from simple perfect fluid or polytropic models. We quote from [25]: "... models satisfying a closed equation of state are relatively few and essentially restricted to dust or linear barotropic models. However, it is worth stressing the importance of considering general equations of state in realistic models, describing for instance, very high density regimes for white dwarfs and neutron stars." Layers with physically realistic equations of state are important in studying the collapse scenarios that result in objects like neutron stars.

In the next section we describe the time development of the radial function, $R(\tau)$, in the exterior Schwarzschild metric. The stress-energy, the equation of state of the layer and its symmetries are discussed in Section III, and we close with a Discussion. Extrinsic curvatures are computed in an Appendix. 


\section{LAYER GEOMETRY}

\section{A. Bounding metrics}

The $2+1$ layer is bounded by two metrics

$$
d s^{2}=g_{a b \pm} d x^{a} d x^{b}=-f_{ \pm} d t^{2}+\left(1 / f_{ \pm}\right) d r^{2}+r^{2} d \Omega^{2}
$$

with + /- denoting exterior/interior.

$$
\begin{aligned}
& f_{+}=1-2 m_{0} / r \\
& f_{-}=1-2 M / r .
\end{aligned}
$$

The metrics correspond to an $m_{0}$ Schwarschild exterior and an $M=$ const Schwarzschild interior. The layer is described by $r=R(\tau)$ and $t=T_{ \pm}(\tau)$. The velocity of the layer as seen by observers moving with the layer in the bounding spaces is $U_{ \pm}^{a}=\left(\dot{T}_{ \pm}, \dot{R}, 0,0\right)$, with corresponding normal vector $n_{a \pm}=\left(-\dot{R}, \dot{T}_{ \pm}, 0,0\right)[4]$. The normal vector is chosen to be outward pointing, toward the exterior spacetime. The velocity normalization imposes the condition

$$
f_{ \pm}(R) \dot{T}_{ \pm}^{2}-\frac{\dot{R}^{2}}{f_{ \pm}(R)}=1 .
$$

This normalization is used to define function $\beta_{ \pm}$

$$
\beta_{ \pm}^{2}:=f_{ \pm}^{2}(R) \dot{T}_{ \pm}^{2}=f_{ \pm}(R)+\dot{R}^{2} .
$$

$\beta_{ \pm}$is chosen positive with $\dot{T}_{ \pm}>0$ and $\dot{R}<0$ describing an infalling layer. An expanding layer can be described by proper time inversion. The equation of state of the layer will not depend on the direction of motion. The induced metric on the layer is

$$
h_{i j \pm}:=\frac{\partial x^{a}}{\partial x^{i}} \frac{\partial x^{b}}{\partial x^{j}} g_{a b \pm}
$$

with

$$
h_{\tau \tau \pm}=-\left(\frac{\partial T_{ \pm}}{\partial \tau}\right)^{2} f_{ \pm}+\left(\frac{\partial R}{\partial \tau}\right)^{2} \frac{1}{f_{ \pm}} .
$$

$h_{\tau \tau \pm}=-1$ from the velocity normalization. The metric of the layer from both sides of the boundary is given by Eq.(1).

In the next section, $R(\tau)$ will be fixed by the requirement of geodesic layer motion in the exterior spacetime. Since both sides of the layer will agree on the layer metric, this will determine the interior radial function. 


\section{B. Exterior geodesics}

For Schwarzschild geodesics in the exterior spacetime we have

$$
\begin{aligned}
\frac{d R}{d \tau} & = \pm \sqrt{E_{0}^{2}-1+2 m_{0} / R} \\
\frac{d T_{+}}{d \tau} & =\frac{E_{0}}{1-2 m_{0} / R}
\end{aligned}
$$

where $E_{0}$ describes the initial point of the layer motion. $E_{0}=1, d R / d \tau<1$ corresponds to a layer beginning its inward drop from infinity. The relations to use in describing the infalling layer from the exterior spacetime are

$$
\begin{aligned}
\frac{d R}{d \tau} & =-\sqrt{2 m_{0} / R} \\
\frac{d T_{+}}{d \tau} & =\frac{1}{1-2 m_{0} / R} .
\end{aligned}
$$

Integrating, one finds

$$
R(\tau)=\left(2 m_{0}\right)^{1 / 3}\left[(3 / 2)\left(c_{1}-\tau\right)\right]^{2 / 3},
$$

which completely determines the layer dynamics. The metric of the layer is

$$
d s^{2}=-d \tau^{2}+\left[(3 / 2) \sqrt{2 m_{0}}\right]^{4 / 3}\left(c_{1}-\tau\right)^{4 / 3} d \Omega^{2} .
$$

\section{LAYER STRESS-ENERGY}

\section{A. Fluid description}

We assume a perfect fluid stress-energy for the layer. The co-moving velocity is $U^{i}=$ $(1,0,0)$ with density $\sigma$ and stress-energy

$$
S_{j}^{i}:=(\sigma+P) U^{i} U_{j}+P h^{i}{ }_{j} .
$$

The stress-energy of the $2+1$ layer is related to jumps in the extrinsic curvatures [1],[2] of the bounding metrics

$$
\begin{aligned}
& -8 \pi S_{j}^{i}:=<K_{j}^{i}>-<K>h_{j}^{i} \\
& -8 \pi S_{\tau}^{\tau}=-<K_{\theta}^{\theta}+K_{\phi}^{\phi}> \\
& -8 \pi S_{\theta}^{\theta}=-<K_{\phi}^{\phi}+K_{\tau}^{\tau}> \\
& -8 \pi S_{\phi}^{\phi}=-<K_{\theta}^{\theta}+K_{\tau}^{\tau}>,
\end{aligned}
$$


where $h_{i j}=\left[-1, R^{2}, R^{2} \sin ^{2} \theta\right], h_{j}^{i}=\delta_{j}^{i}$ and where

$$
<K_{j}^{i}>:=K_{j+}^{i}-K_{j-}^{i}
$$

is the jump in the extrinsic curvature and $K:=K_{i}^{i}$. The extrinsic curvatures are worked out in Appendix A. For the constant mass exterior Schwarzschild metric they are

$$
\begin{aligned}
& K_{\theta+}^{\theta}=K_{\phi+}^{\phi}=1 / R \\
& K_{\tau+}^{\tau}=0 .
\end{aligned}
$$

In the interior, with mass parameter $M$, the extrinsic curvatures are

$$
\begin{aligned}
& K_{\theta-}^{\theta}=K_{\phi-}^{\phi}=\beta_{-} / R \\
& K_{\tau-}^{\tau}=\frac{M-m_{0}}{R^{2} \beta_{-}} .
\end{aligned}
$$

The layer density is

$$
4 \pi \sigma=-<K_{\theta}^{\theta}>=\frac{\beta_{-}-1}{R}
$$

where

$$
\beta_{-}=\sqrt{1+2 \frac{m_{0}-M}{R}} .
$$

For $R>0$, the range of $\beta_{-}$is restricted to $\beta_{-}>1$ for positive layer density. This requires $m_{0}>M$. The stress is

$$
\begin{aligned}
& 8 \pi P=\left\langle K_{\theta}^{\theta}+K_{\tau}^{\tau}>=\frac{-\beta_{-}+1}{R}+\frac{m_{0}-M}{R^{2} \beta_{-}}\right. \\
& 8 \pi P=-\frac{\left(\beta_{-}-1\right)^{2}}{2 \beta_{-}} \frac{1}{R} .
\end{aligned}
$$

A layer mass $m_{L}$ can be calculated from the layer density

$$
\begin{aligned}
& m_{L}=4 \pi \sigma R^{2} \\
& m_{L}=\frac{2\left(m_{0}-M\right)}{\beta_{-}+1} .
\end{aligned}
$$

The physical picture has an exterior Schwarzschild metric with mass parameter $m_{0}$ formed from the stress-energy of the layer plus the interior Schwarzschild metric with mass $M$. The binding energy of the layer contributes to the value of $m_{0}$, as can be seen by eliminating $\beta_{-}$ between equations (22) and (24):

$$
m_{L}=m_{0}-M-\frac{m_{L}^{2}}{2 R} .
$$


The layer mass is not constant due to work done by the stress

$$
\frac{d\left(\sigma R^{2}\right)}{d \tau}=-P \frac{d R^{2}}{d \tau}
$$

This has also been noted by Carr and Yahil [39] for a self-similar Friedman universe and by Visser [40] in dynamic wormholes.

\section{B. Equation of State}

The relation between the density and stress for Lemaitre layers is

$$
P=-\sigma \frac{\beta_{-}-1}{4 \beta_{-}}
$$

or

$$
\beta_{-}=\frac{1}{1+4 P / \sigma}
$$

Expression (28) can be rewritten as

$$
\beta_{-}-1=-\frac{4 P / \sigma}{1+4 P / \sigma}, \quad \beta_{-}+1=\frac{2+4 P / \sigma}{1+4 P / \sigma}
$$

or

$$
\beta_{-}^{2}-1=-8(P / \sigma) \frac{1+2 P / \sigma}{(1+4 P / \sigma)^{2}} .
$$

Eliminating $R$ between Equations (21) and (22) allows $\sigma$ to be expressed as

$$
\sigma=\frac{\left(\beta_{-}-1\right)\left(\beta_{-}^{2}-1\right)}{8 \pi\left(m_{0}-M\right)} .
$$

Substituting for $\left(\beta_{-}-1\right)$ and $\left(\beta_{-}^{2}-1\right)$ in terms of $P / \sigma$ above, yields the equation of state for the Lemaitre layer

$$
P^{2}=\left[\frac{\pi\left(m_{0}-M\right)}{4}\right] \frac{\sigma^{3}(1+4 P / \sigma)^{3}}{(1+2 P / \sigma)} .
$$

This expression can be rewritten for the density or stress in terms of the ratio $x:=P / \sigma$.

$$
\sigma=\frac{4 x^{2}(1+2 x)}{\pi\left(m_{0}-M\right)(1+4 x)^{3}} .
$$

For a static membrane under tension, $x$ is the negative of the sound speed squared, $\partial P / \partial \sigma$. The ' $x$ ' ranges of possible interest are

$$
\begin{gathered}
I: 0 \leq x \\
I I:-1 / 4 \leq x \leq 0 \\
I I I:-1 / 2<x \leq-1 / 4 \\
I V:-1 \leq x<-1 / 2
\end{gathered}
$$


The relative size of $m_{0}$ to $M$ provides three mass regions to consider. $m_{0}=M$ has no extrinsic curvature jumps and no layer. For $m_{0}>M, \beta_{-}>1$ is required for positive layer density. This eliminates the positive range of $x . \beta_{-}>0$ requires $-1 / 4 \leq x$ and this eliminates the third and fourth region. Region II is the only allowed range and this sets a new limit on the stress/density ratio for layers collapsing in a Schwarzschild geometry. Figure 1 shows the variation of the stress and density in Region II. A portion of Region I has been included for clarity.

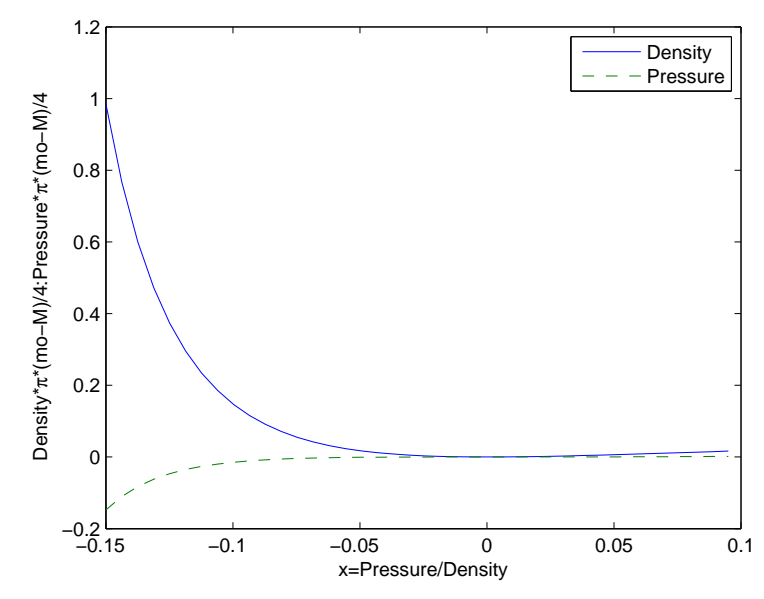

FIG. 1: Scaled density and pressure vs pressure/density

In general the equation of state does not have any of the usual simple forms. It is a cubic equation in pressure and, depending on the value of $\sigma$, can contain simpler equations of state in the root structure. Rewriting Eq.(29), one finds

$$
\begin{aligned}
& \bar{p}:=p(\pi / 4)\left(m_{0}-M\right) \\
& \bar{\sigma}:=\sigma(\pi / 4)\left(m_{0}-M\right) \\
& \bar{p}^{3}(64 \bar{\sigma}-2)+\bar{p}^{2}\left(48 \bar{\sigma}^{2}-\bar{\sigma}\right)+12 \bar{\sigma}^{3} \bar{p}+\bar{\sigma}^{4}=0
\end{aligned}
$$

The equation of state, Eq.(32), is plotted in Figure 2 showing the regions where, for a single density value, there are three real roots, two real roots, and one real root giving associated values of the pressure. The negative pressure region corresponds to the layer tension. In this region, for small density, there are two possible tensions for each value of density while for larger density, there is a 1-1 non-linear relationship. 


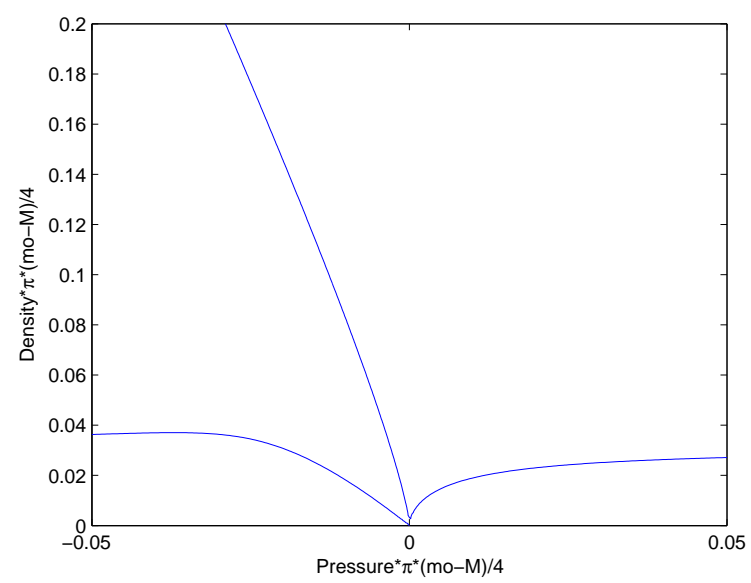

FIG. 2: Scaled density vs scaled pressure for the equation of state

Expanding Eq.(29) one finds

$$
P \approx \frac{4 x^{3}}{\pi\left(m_{0}-M\right)}(1-10 x+\ldots)
$$

For $x<<1$ the equation of state appears approximately polytropic with index 2 [41]. Layers are most commonly described as perfect fluids or scalar fields. Within the perfect fluid models, several EOS [25],[38],[42] have been suggested to analyze the layer stress energy content. A commonly discussed form is

$$
P=-n \sigma
$$

which plays a crucial role in $3+1$ homothetic solutions [43]. The layer is dropping on a Schwarzschld/Lemaitre geodesic. The actual equation of state does not change but it can be modeled with simpler forms having time dependent parameters. This is a different point of view than many dynamic layer treatments which assume a static equation of state and let that drive the layer dynamics. For example, Eq.(27) for the Lemaitre layer implies that $n(\tau)$ changes as the layer drops with the $\mathrm{n}$ value given by

$$
n(\tau)=-x=\frac{\beta_{-}-1}{4 \beta_{-}} .
$$

$n(\tau)$ runs through the range $0<n<1 / 4$ for the positive density layer. We note that $x=0$ is the dust solution corresponding to $m_{0}=M$, the vanishing layer condition, or to very large $R$. Positive $\beta_{-}>1$ describes an inward moving layer with positive layer density, mass 
and tension. The constraint $m_{0}>M$ implies that $r=M$ is inside the $r=2 m_{0}$ trapped surface. The physical picture of the layer dropping inward toward an interior object of mass $M$ implies that the layer will cross the $2 m_{0}$ horizon. The value of $x$ at the horizon, $x_{H}$, is parameterized by the ratio $M / m_{0}$

$$
4 x_{H}=\frac{1}{\sqrt{2-M / m_{0}}}-1 .
$$

The maximum value of $x_{H}, x_{H} \sim-0.07$, occurs for a Minkowski, $M=0$ interior, with $x_{H}$ decreasing as the size of the interior $M$ approaches $m_{0}$. With $M=0$, the tension of the layer is $P=-.07 \sigma$ at $R=2 m_{0}$, increasing inward to $-1 / 4$ at the $R=0$ singularity. The layer mass at $R=2 m_{0}$ is less than $m_{0}$, since $m_{0}$ will include the layer binding energy.

The region, $m_{0}<M$, describes a layer with negative density and mass and with $\beta_{-}<1$, with $x$ positive. For this case $R=2 m_{0}$ lies inside $R=2 M$. From Eq.(22), $\beta_{-}=0$ at $R=2\left(M-m_{0}\right)$. At this radius, the layer has infinite tension. The interior Schwarzschild solution could be the exterior of a third core fluid region with a boundary at $R>2 \mathrm{M}$, eliminating trapped surfaces from the vacuum motion.

Another indication of the complexity of the Lemaitre layer is the sound speed,

$$
V_{s}^{2}:=\frac{\partial P}{\partial \sigma}
$$

Using the equation of state, this is

$$
V_{s}^{2}=\frac{x}{2(1+x)}\left(3+8 x+8 x^{2}\right) .
$$

$V_{s}^{2}$ is negative over the range $-1 / 4<x<0$ indicating instabilities in the layer [44].

\section{DISCUSSION}

In this paper we have discussed the geodesic collapse of an Israel layer in an exterior Schwarzschild metric. The geodesic drop of the layer determines the dynamic development of the layer's motion. Although their geodesic motion is simple, Lemaitre layers are physically interesting structures because of their complex equation of state, which can approximate some of the simpler equations of state commonly used in layer dynamics. Some of the simplest equations of state can be described by time dependent models of the actual stress density relation. A barotropic equation of state is often used to describe Israel layers, for 
example in effective potential treaments [26],[45],[46] and discussion of bubble structures [47]. In the simple model considered here, the layer can have a time dependent barotropic relation, $P=-n(\tau) \sigma$. It has an $R \rightarrow 0$ limiting value $P=-\sigma / 4$, with $n(\tau)$ still very small as the layer reaches the $R=2 m_{0}$ surface.

The range of $P / \sigma$ for positive density, has a lower bound of $-1 / 4$. This is a new restriction on the range of the stress/density ratio for collapsing layers. Garfinkle and Vuille [26] considered the layer between two Schwarzschild metrics with no motion restrictions. The value $P / \sigma=-1 / 2$ was identified as a boundary for their effective potential [45], [46] to have stationary points. The effective potential for the Lemaitre layer is simple with no stationary points or turning points and the boundary $P=-\sigma / 4$ occurs at the point $R=0$.

Fluids collapsing along geodesics in a Schwarzschild metric have been considered by Lynden-Bell and Lemos [48], generalizing the description of a Newtonian cold gas collapsing from rest at infinity [49]. The collapse of the Lemaitre layer and the cold gas have some similarities. They are both described by the the same $R(\tau)$ and for either case Eq.(12) can be written as

$$
\frac{4 m_{0}}{3}\left[\frac{R_{i}(\tau)}{2 m_{0}}\right]^{3 / 2}=\tau_{c}-\tau_{i}
$$

where $\tau_{i}$ is the proper time to reach $R_{i}$ and $\tau_{c}$ is the time to reach $R=0$. In the less limiting case of a Newtonian sphere with interior mass $m$, Lynden-Bell and Lemos show that $\tau_{c}$ can be written as a function of the mass

$$
\tau_{c}=B m^{b}+\tau_{0}
$$

where $\tau_{0}$ is a constant of integration. Generalizing this relation to Einstein's gravity, $b=1$ is identified as a value of special interest corresponding to metric homothetic symmetry. For the Lemaitre layer, $\tau_{c}$ is just the constant $c_{1}=\tau_{0}$, corresponding to $B=0$ in the cold gas solution. $\tau_{c}$ is related to the time $\tau_{H}$ for a layer to reach the $R=2 m_{0}$ surface by

$$
\tau_{c}=4 m_{0} / 3+\tau_{H}
$$

The Lemaitre layer can be considered as a special member of the $b=1, B=0$ geodesic solutions which include cold gas collapse. One also notes that the exterior bounding Lemaitre metric exhibits second kind kinematic similarity [50], reflected by $R(\tau)$ in Eq.(12), which is transferred to the layer and maintained thoughout the motion. 
There is growing interest in Israel layers as objects of research rather than an aid in matching metrics. The increasing interest in expanding the physical properties of Israel layers has led to descriptions incorporating layer parameters like shell thickness [36], [37] and surface packing fraction [13]. While Israel layers are still linked to the properties of the two bounding spaces, even simple layers such as the Lemaitre layer considered here, require more physical content than the density and pressure coming from simple perfect fluid or polytropic models. The time dependent models for the actual stress density relations described here, represent one way these simple models can still be used effectively and related to the literature using simple equations of state.

\section{APPENDIX A: EXTRINSIC CURVATURES}

The stress-energy of the layer is determined by jumps in the extrinsic curvatures, $K_{a b}$ of the bounding metrics across the Israel layer. The bounding metrics considered here are

$$
d s^{2}=-f_{ \pm} d t^{2}+\left(1 / f_{ \pm}\right) d r^{2}+r^{2} d \Omega^{2}
$$

with $\left(f_{+}, f_{-}\right)=\left(1-2 m_{0} / r, 1-2 M / r\right)$. The layer is described by $r=R(\tau)$ and $t=$ $T_{ \pm}(\tau)$. The velocity of the layer as seen by observers moving with the layer in the bounding spaces is $U_{ \pm}^{i}=\left(\dot{T}_{ \pm}, \dot{R}, 0,0\right)$, with corresponding outward pointing normal vector $n_{a \pm}=$ $\left(-\dot{R}, \dot{T}_{ \pm}, 0,0\right)[4]$. The velocity normalization imposes

$$
f_{ \pm}(R) \dot{T}_{ \pm}^{2}-\frac{\dot{R}^{2}}{f_{ \pm}(R)}=1
$$

The normalization is used to define a function, $\beta_{ \pm}$

$$
\beta_{ \pm}^{2}:=f_{ \pm}^{2}(R) \dot{T}_{ \pm}^{2}=f_{ \pm}(R)+\dot{R}^{2}
$$

We choose $\beta_{ \pm}>0$. $\beta_{ \pm}<0$ corresponds to $\tau \rightarrow-\tau$. The metric of the layer from both sides of the boundary is

$$
d s^{2}=-d \tau^{2}+R^{2}(\tau) d \Omega^{2}
$$

The extrinsic curvature is defined in terms of the normal to the layer, $n_{a}$ and the projection operator onto the layer, $h_{a b}$ as

$$
K_{i j}:=n_{a ; b} h_{i}^{a} h_{j}^{b}
$$


The two extrinsic curvatures are $K_{\theta}^{\theta}$ and $K_{\tau}^{\tau} \cdot K_{\theta}^{\theta}$ is easily calculated from the definition and one finds

$$
K_{\theta \pm}^{\theta}=\frac{\beta_{-}}{R}=\frac{\sqrt{f_{ \pm}(R)+\dot{R}^{2}}}{R} .
$$

where we use $\beta_{-}$to indicate the range of $\beta_{ \pm}$to be used. Using the infalling geodesic value for $\dot{R}=-\sqrt{2 m_{0} / R}$ we have

$$
K_{\theta \pm}^{\theta}=\frac{\beta_{-}}{R}=\frac{\sqrt{f_{ \pm}(R)+2 m_{0} / R}}{R}
$$

$K_{\tau}^{\tau}$ is more easily calculated from the acceleration $K_{\tau}^{\tau}=n^{i} a_{i}$. The acceleration vector is

$$
a^{i}=\left[\ddot{T}+\frac{\dot{T} \dot{f}}{f}, \ddot{R}+\frac{\dot{f}}{2 \dot{R}}, 0,0\right] .
$$

Dotting with the normal and using the definition of $\beta_{ \pm}$, one finds

$$
K_{\tau \pm}^{\tau}=\frac{\dot{\beta}_{ \pm}}{\dot{R}}
$$

All cases will have the same exterior functions. In the interior, the extrinsic curvatures are

$$
\begin{aligned}
\beta_{-} & =\sqrt{1+\frac{2\left(m_{0}-M\right)}{R}} \\
K_{\theta-}^{\theta} & =\beta_{-} / R \\
K_{\tau-}^{\tau} & =\frac{M-m_{0}}{R^{2} \beta_{-}} .
\end{aligned}
$$

[1] W. Israel, Nuov. Cim. 44B, 1 (1966).

[2] W. Israel, Nuov. Cim. 48B, 463 (1967).

[3] C. Barrabes and W. Israel, Phys. Rev. D 43, 1129 (1991).

[4] E. Poisson, A Relativist's Toolkit (Cambridge University Press, Cambridge, England, 2004) p.93.

[5] V. de la Cruz and W. Israel, Phys. Rev. 170, 1187 (1968).

[6] P. Musgrave and K. Lake, Classical Quantum Gravity 14, 1285 (1997).

[7] C. Barrabes and P. A. Hogan, Phys. Rev. D 58, 044013 (1998).

[8] V.A. Berezin, V.A. Kuzmin and I.I. Trachev, Phys. Rev. D 36, 2919 (1987).

[9] S. Ansoldi, Classical Quantum Gravity 19, 6321 (2002). 
[10] S. Ansoldi, arXiv:gr-qc/0701082. ( To appear in the proceedings of the Eleventh Marcel Grossmann Meeting on General Relativity, July 23-29, 2006, Freie Universitaet Berlin, Berlin, $G R$

[11] L. Ortiz and M.P. Ryan, Jr, Gen. Relativ. Gravit. 39, 1087 (2007).

[12] M. Ambrus and P. Jahicek, Phys. Rev. D 72, 064025 (2005).

[13] J.P. Krisch, J. Math. Phys. (N.Y.) 46, 042506 (2005).

[14] S.Bayin, E.N. Glass and J.P. Krisch, J. Math. Phys. (N.Y.) 47, 012501 (2006).

[15] J.P. Krisch, J. Math. Phys. (N.Y.) 47, 122501 (2006).

[16] E. Gravanis and S. Willison, Phys. Rev. D 75, 084025 (2007).

[17] F. Rahaman, M. Kalam and S. Chakraborti, arXiv:gr-qc/0611134. (Int. J. Mod. Phys. D, to be published)

[18] E.F. Eiroa and C. Simeone, Phys. Rev. D 71, 127501 (2005).

[19] C. Barrabes and W. Israel, Phys. Rev. D 71, 064008 (2005).

[20] F.S.N. Lobo and P. Crawford, Classical Quantum Gravity 22,.4869 (2005).

[21] M. Mars and J.M.M. Senovilla, Classical Quantum Gravity 10, 1865 (1993).

[22] R. Vera, Classical Quantum Gravity 19, 5249 (2002).

[23] M. Mars, Classical Quantum Gravity 22, 3325 (2005).

[24] M. Mars, F. C. Mena and R. Vera, arXiv/gr-qc/07040078.

[25] J. Kijowski, G. Magli and D. Malafarina, Gen. Relativ. Gravit. 38, 1697 (2006).

[26] D. Garfinkle and C. Vuille, Classical Quantum Gravity 6, 1819 (1989).

[27] P.D. Lasky and A.W.C. Lun, Phys. Rev. D 75, 104010 (2007).

[28] P.O. Mazur and E. Mottola, arXiv:gr-qc/0109035.

[29] P.O. Mazur and E. Mottola, Proc. Nat. Acad. Sci. 111, 9545 (2004).

[30] M. Visser and D.L. Wiltshsire, Classical Quantum Gravity 21, 1135 (2004).

[31] B. Carter, Classical Quantum Gravity 22, 4551 (2005).

[32] C. Cattoen, T. Faber and M. Visser, Classical Quantum Gravity 22, 4189 (2005).

[33] F.S.N. Lobo and A.V.B. Arellano, Classical Quantum Gravity 24, 1069 (2007).

[34] A. deBenedictis, D. Horvat, S. Ilijic, S. Kloster and K.S.Viswanathan, Classical Quantum Gravity 23, 2303 (2006).

[35] A. E. Broderick and R. Narayan, Classical Quantum Gravity 24, 659 (2007).

[36] S. Khakshournia and R. Mansouri, Gen. Relativ. Gravit. 34, 1847 (2002). 
[37] Sh. Khosravi, S. Khakshournia and R. Mansouri, Classical Quantum Gravity 23, 5927 (2006).

[38] H. Maeda, T. Harada and H. Iguchi, Phys. Rev.D 66, 27501 (2002).

[39] B.J. Carr and A. Yahil, Astrophys. J. 360, 330 (1990).

[40] M. Visser, Lorentzian wormholes: From Einstein to Hawking, (AIP Press, New York, 1995) p.183

[41] S. Chandrasekhar, An Introduction to the Study of Stellar Structure, (Dover, New York, 1957), Ch. 6.

[42] F.S.N. Lobo, Phys. Rev. D 75, 024023 (2007).

[43] M.E. Cahill and A.H. Taub, Comm. Math. Phys. 21, 1 (1971).

[44] E.W. Kolb and M.S. Turner, The Early Universe, (Addison-Wesley, MA, 1990), p. 342.

[45] K. Lake, Phys. Rev. D 19, 2847 (1979).

[46] K. Lake and R. Wevrick, Canadian Journal of Physics 64, 165 (1986).

[47] J.R. Ipser, Phys. Rev. D 36, 1933 (1987).

[48] D. Lynden-Bell and J.P.S. Lemos, MNRAS 233, 197 (1988).

[49] M.V. Penston, MNRAS 144, 425 (1969).

[50] B. Carter and R.N. Henriksen, Ann. Physique Supp. 14, 47 (1989). 